\title{
Characterization of Collagenase Blend Enzymes for Human Islet Transplantation
}

\author{
Barbara Antonioli, ${ }^{1}$ Isabella Fermo, ${ }^{2}$ Silvia Cainarca, ${ }^{3}$ Simona Marzorati, ${ }^{1}$ Rita Nano, ${ }^{1}$ \\ Michela Baldissera, ${ }^{1}$ Angela Bachi, ${ }^{4}$ Rita Paroni, ${ }^{5}$ Camillo Ricordi, ${ }^{6}$ and Federico Bertuzzi ${ }^{1,7,8}$
}

\begin{abstract}
Background. Efficient islet isolation represents a necessary requirement for successful islet transplantation as a treatment for type 1 diabetes. The choice of collagenase for pancreas digestion is critical for the isolation outcome, and Liberase $^{\mathrm{TM}}$ is the most widely used enzyme, although large intra-batched variability in activity and efficiency has been observed.

Methods. The aim of this study was to characterize Liberase ${ }^{\mathrm{TM}}$ components and their relative role in pancreas digestion. Liberase batches were characterized by microelectrophoresis.

Results. By means of microelectrophoresis, we identified three main proteins each with different prevalences between batches. Two proteins were found to correspond to class I (CI) and one to class II (CII) collagenase. In a series of 163 islet isolations, we observed that the CII correlated with islet yield $(P<0.001)$ and digestion time $(P<0.001)$; additionally, $\mathrm{CI}$ directly correlated with purity $(P=0.028)$. Finally, when $\mathrm{CII}$ and one of the $\mathrm{CI}$ isoforms were $>50$ percentile, 15 of 36 preparations were transplanted, with 27 of 127 transplanted in the other cases $(P=0.013)$.

Conclusion. These results represent an important step toward the characterization of enzymes, with the final aim of identifying key components for a standardized product.
\end{abstract}

Keywords: Islet isolation, Purification, Collagenase, Proteases, Islet transplantation.

(Transplantation 2007;84: 1568-1575)

A standardization of human islet isolation that produces a sufficient number of good-quality islets in a reproducible way is an essential requisite for a successful clinical transplant program (1). An optimization of isolation techniques would permit an increased number of transplants and a reduction of costs, which are important requisites for securing reimbursement for this procedure from health insurance providers and national health systems.

To date, a real standardized procedure has not been possible because of the fact that one of the crucial steps of

\footnotetext{
${ }^{1}$ Unit of Cell Therapy for Type 1 Diabetes Mellitus, San Raffaele Scientific Institute, Milan, Italy.

${ }^{2}$ Separative Techniques Unit, San Raffaele Scientific Institute, Milan, Italy.

${ }^{3}$ Axxam Srl, San Raffaele Biochemical Science Park, Milan, Italy.

${ }^{4}$ Biological Mass Spectrometry, DIBIT, San Raffaele Scientific Institute, Milan, Italy.

${ }^{5}$ Lab. Analytical and Medical Chemistry, University of Milan, S. Paolo Hospital, Milan, Italy.

${ }^{6}$ Diabetes Research Institute, University of Miami, Miami, FL.

${ }^{7}$ Medicine Department, ISMETT, 90127 Palermo, Italy.

${ }^{8}$ Address correspondence to: Federico Bertuzzi, M.D., Unit of Cell Therapy for Type 1 Diabetes, S. Raffaele Scientific Institute, Via Olgettina 60, Milan and, Medicine Department, Mediterranean Institute for Transplantation and Advanced Specialized Therapies. Via Tricomi 1, Palermo, Italy.
}

E-mail: fbertuzzi@ISMETT.edu

Received 16 July 2007. Revision requested 4 September.

Accepted 14 September 2007.

Copyright $\odot 2007$ by Lippincott Williams \& Wilkins

ISSN 0041-1337/07/8412-1568

DOI: $10.1097 / 01 . t p .0000295719 .88525 .60$ isolation, organ digestion, does not yield a standardized product. Second, the enzyme used, collagenase, presents different characteristics from batch to batch, and from vial to vial, because of problems of instability $(2,3)$. Furthermore, there are no precise parameters for the evaluation of enzymatic efficacy before its use; therefore, it is necessary to experiment with an enzyme batch many times before determining its suitability. Enzyme activity per se, commonly available for each batch, is not considered a useful parameter for the selection of successful batches and therefore other parameters or methods of analyses are expected at this purpose (4).

The introduction of purified products has not solved that problem (5-6), because the proposed enzymes are always obtained from Clostridium histolyticum cultures and remain different from batch to batch as well. A significant variability of enzyme activity was recently described not only between Liberase batches but also between vials of the same batch (3). The first experiments with a type of recombinant collagenase have not provided additional information (7). In fact, with the data now available, standardization remains a difficult challenge, because the composition of efficient enzyme blends, especially for human pancreas digestion, is not yet well defined ( 8 ).

Different isoforms of collagenase, identified by the Greek letters $(\alpha, \beta, \gamma, \delta, \varepsilon, \zeta)$, belong to two classes (CI and CII) (9-12). Concerning the rat pancreas digestion, it has been reported that the CII is the most efficient at digesting 
exocrine tissue, whereas $\mathrm{CI}$ increases the digestion rate (13, 14); therefore, an appropriate mix of CI and CII has been proposed to optimize islet isolation (15-18). In the early stages of digestion, neutral proteases also are needed, but in excessive amounts these can destroy islet integrity (19-21). A protocol for using CI and CII together with neutral proteases for the human pancreas digestion has been proposed (22), but nothing is known about the role of the different isoforms of collagenase within the same class.

\section{MATERIALS AND IMETHODS}

\section{Islet Isolation}

Islets were isolated using the automated method described by Ricordi (23) and purified as previously described (24). The enzyme used for isolation was Liberase ${ }^{\mathrm{TM}}(0.5-1.4$ $\mathrm{mg} / \mathrm{mL}$; Roche, Indianapolis, IN). In $350 \mathrm{~mL}$ of Hank's solution, an amount of enzymes ranging from 175 to $500 \mathrm{mg}$ (related to the trimmed pancreas weight, about $4 \mathrm{mg}$ of enzyme per gram of pancreas) was gentle dissolved. The enzyme concentration was further reduced (up to $50 \%$ ) if the donor was younger than 20 years old. All preparations were submitted to the purification (for transplantation or for research purpose) with the exception of those in which digestion completely failed (completely destroyed islets or undigested tissue). In these cases, the number of isolated islets was reported to be 0 , as well as in those isolations in which the purification completely failed and all the tissue was discharged. The final number of isolated islets considered in the analyses and their corresponding purity were calculated, including all gradient layers with islets up to remaining under $10 \mathrm{~mL}$ of total tissue volume (as in the case of a possible islet transplantation). Islet cell viability was estimated by propidium iodide staining and insulin responsiveness to glucose by static incubation, as previously described (4).

\section{Bacterial Collagenases}

Liberase $^{\mathrm{TM}} \mathrm{HI}$ (15 batches) from C. histolyticum used in human isolation were analyzed. The different sample batches were collected during a period of 3 years. Each aliquot of Liberase ${ }^{\mathrm{TM}}$ was collected before enzyme reconstitution from a new vial, after proper equilibration on ice and mixing of the vial contents (3). Four samples were reconstituted from each batch with $10 \mathrm{mM}$ EDTA solution (Sigma-Aldrich Corporation, S. Louis, MO) on ice and quickly loaded for characterization using different analyses. A protocol for the preparation of enzyme samples was tested and standardized to prevent uncontrolled degradation prior to biochemical analysis and to be used in isolations. The samples' final concentration was $1 \mathrm{mg} / \mathrm{ml}$ for the Protein 200 Plus Assay (Agilent Technologies, Palo Alto, CA; milligram of powder mass provided by the manufacture).

The samples from lyophilized separated components, Collagenase Class I, Collagenase Class II, and Thermolysin (Roche, Indianapolis, IN), were similarly collected and reconstituted. Activity is expressed in Wunsch units (U/mg or U/vial) for collagenase class II and Liberase, in Caseinase units (U/mg or U/vial) for neutral proteases. These values were obtained from the Roche's Certificate of Analysis.

\section{Microelectrophoresis Analysis}

The Protein 200 Plus assay (Agilent Technologies, Palo Alto, CA) allows sizing of proteins ranging from 14 to 200 $\mathrm{kDa}$. Sensitivity of Protein 200 Plus assay is $20 \mathrm{ng} / \mu \mathrm{l}$ bovine serum albumin (BSA) in phosphate-buffered solution (PBS). The chip-based separations were performed on the Agilent 2100 Bioanalyzer in combination with the Protein 200 LabChip kit and the dedicated Protein 200 assay software (Agilent Technologies, Palo Alto, CA); each sample was double loaded. Data are displayed as electropherogram or gel-like image; a table with information regarding peaks (migration time, molecular weight, peak area, \% total) is generated for each sample. The three main peaks were defined G, D, and B. The proportion of the peak area of a specific collagenase fraction relative to the sum of all peaks was expressed as a ratio, and reported as an "Index" (3). To evaluate the intra-assay coefficient of variations $(\% \mathrm{CV})$, samples from the same vial were analyzed on the same microchip. The $\mathrm{CV}$ values were $2.6 \%, 5.1 \%$, and $1.7 \%$ for peaks $\mathrm{G}, \mathrm{D}$, and $\mathrm{B}$ respectively. To
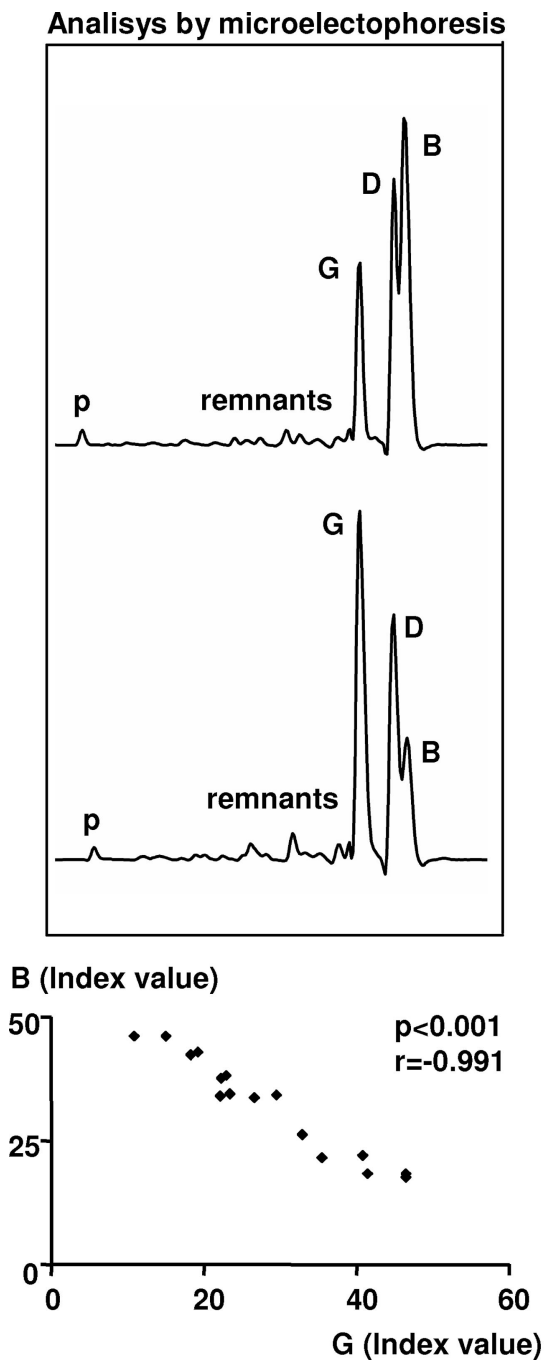

b)

FIGURE 1. Liberase analyses by microelectrophoresis. (a) Liberase ${ }^{\mathrm{TM}}$ profiles in analysis by microelectrophoresis of two different batches (93017120, 93047420). Peak B and peak $\mathrm{G}$ are inversely correlated (b). 
evaluate differences between vials of the same batch, samples from different vials were tested on the same microchip (intralot $\% \mathrm{CV}$ ). The CV values were $2.4 \%, 0.7 \%$, and $5.3 \%$ for peaks $G, D$, and B, respectively. Samples from different vials of the same lot were analyzed using different microchips at different times: $6.6 \%, 6.7 \%, 5.5 \%$ were the $\mathrm{CV}$ values founded for peaks $\mathrm{G}, \mathrm{D}$, and $\mathrm{B}$ respectively.

\section{Islet Transplantation}

Transplantation was performed between 2 and $72 \mathrm{hr}$ after isolation. Preparations were considered adequate for transplantation according to the following criteria: (1) NE ( $>5,000$ per $\mathrm{kg}$ body weight); (2) purity $>20 \%$ (dithizonestaining determination of islet/total mass); and (3) islet sterility and cell viability $>70 \%$ analyzed by propidium iodide (4). Purification was defined to be $20 \%$ or greater because, in our experience, this limit maintains the tissue volume for transplant to less than $10 \mathrm{~mL}$. A total of 42 islet preparations were transplanted into HLA-mismatched recipients affected by type 1 diabetes as previously described (4). Recipients gave informed consent and our investigations were approved by the responsible ethics committee (institutional review board). Studies were conducted in accordance with the Declaration of Helsinki as revised in 2000 (http://www.wma. net/e/policy/b3.htm).

\section{Statistical Analysis}

Data were collected from 163 performed with Liberase ${ }^{\mathrm{TM}}$. Isolations were not consecutive: procedures using collagenase type $\mathrm{P}$ were excluded from the analyses. follows:

Parameters to qualify Liberase components were as

- Isolation outcomes in terms of the total number of islets expressed as final NE (the absolute number corrected for coefficients based on islet volume), digestion time and purity. Final NE was preferred to prepurification NE. The purification method in this panel of isolations was the same and considering final NE instead of prepurification, we included the possible interference of enzyme also on purification.

- Islet cell viability (as percentage of live cells after propidium iodide staining) and insulin responsiveness to glucose, as secretion index (the ratio between basal and stimulated insulin secretion) as previously described (4).

- Decision whether or not to transplant the preparation. Islet preparation is defined suitable for the transplant not only on the basis of the islet number, but also on cell viability and purity (4). Therefore, this represents an overall and indirect parameter of islet preparation quality.

- Transplantation outcome in terms of posttransplant cpeptide levels and exogenous insulin requirement 1 week and 1 month after transplantation. Our recipients often received 2 or more preparations simultaneously or in subsequent transplantation. Also, immunosuppressive treatment should vary in different patients. Therefore, the number of transplants available for this analysis ( single islet preparation transplantation) was limited to 10 patients, with at least 1 month follow-up and similar immunosuppressive treatment (Edmonton protocol, 4).

Variables did not show a Gaussian distribution, and a nonparametric analysis was performed. Where both variables were ordinal values, correlations were performed using the Spearman rank correlation test and expressed as Spearman's correlation coefficient. All comparisons are 2 tailed. When variables were nominal values, a $\chi^{2}$-square test was used and a $P$ value lower than 0.05 was considered to be statistically significant.

TABLE 1. Characteristics of different Liberase ${ }^{\mathrm{TM}}$ batches and prevalences of each peak

\begin{tabular}{|c|c|c|c|c|c|c|c|}
\hline $\begin{array}{l}\text { Liberase }^{\mathrm{TM}} \\
\text { batches }\end{array}$ & $\begin{array}{l}\text { Activity } \\
\text { (U/mg) }\end{array}$ & $\begin{array}{c}\text { Neutral } \\
\text { protease }(\mathrm{U} / \mathrm{mg})\end{array}$ & $\begin{array}{l}\text { Peak p } \\
\text { index }\end{array}$ & $\begin{array}{l}\text { Peak G } \\
\text { index }\end{array}$ & $\begin{array}{l}\text { Peak D } \\
\text { index }\end{array}$ & $\begin{array}{l}\text { Peak B } \\
\text { index }\end{array}$ & $\begin{array}{c}\text { Remnant } \\
\text { index }\end{array}$ \\
\hline 93047420 & 4.248 & 155.6 & 0.013 & 0.466 & 0.292 & 0.173 & 0.056 \\
\hline 90287220 & 4.922 & 193.2 & 0.022 & 0.109 & 0.287 & 0.459 & 0.123 \\
\hline 93017120 & 4.170 & 147.2 & 0.016 & 0.194 & 0.286 & 0.426 & 0.078 \\
\hline 93114320 & 4.216 & 120.7 & 0.018 & 0.230 & 0.284 & 0.378 & 0.090 \\
\hline 93126020 & 4.048 & 120.4 & 0.026 & 0.184 & 0.354 & 0.420 & 0.016 \\
\hline 93145320 & 4.454 & 133.8 & 0.026 & 0.357 & 0.306 & 0.213 & 0.098 \\
\hline 93152720 & 4.970 & 132.1 & 0.024 & 0.296 & 0.310 & 0.340 & 0.030 \\
\hline 93028820 & 4.404 & 146.9 & 0.017 & 0.152 & 0.317 & 0.458 & 0.056 \\
\hline 93045820 & 3.900 & 140.3 & 0.029 & 0.416 & 0.282 & 0.180 & 0.093 \\
\hline 93142020 & 4.794 & 147.6 & 0.024 & 0.331 & 0.286 & 0.260 & 0.099 \\
\hline 93167720 & 5.058 & 142.4 & $-^{a}$ & 0.224 & 0.354 & 0.373 & 0.049 \\
\hline 93163920 & 4.402 & 128.4 & 0.019 & 0.268 & 0.311 & 0.335 & 0.067 \\
\hline 93187520 & 4.226 & 135.2 & 0.027 & 0.223 & 0.336 & 0.336 & 0.078 \\
\hline 93172920 & 4.144 & 140.2 & 0.019 & 0.236 & 0.341 & 0.342 & 0.062 \\
\hline 93273520 & 4.996 & 137.6 & 0.017 & 0.410 & 0.294 & 0.219 & 0.060 \\
\hline
\end{tabular}

Activity is expressed in Wunsch units (U/mg) and neutral proteases in Caseinase units (U/mg). The prevalence of the peak area of a specific collagenase fraction, relative to the sum of all peaks, was expressed as a ratio and reported as "Index."

${ }^{a}$ This batch did not contained neutral protease. 


\section{RESULTS}

\section{Analysis of Protein Content in Liberase ${ }^{\mathrm{TM}}$ Batches by Electrophoresis}

Enzyme blends were first studied by microelectrophoresis. Unexpectedly, differently from that reported by the Roche data sheet for Liberase ${ }^{\mathrm{TM}}$, and similarly to that previously reported by Lakey (3), more than two peaks appeared from the analyses of batches. All Liberase ${ }^{\mathrm{TM}}$ batches showed three main peaks $(G, D, B)$ and one minor one (p) (see Fig. 1a for the profile of two representative batches of those included in the study). The molecular weight of peaks $p, G, D$, and $B$ were $34 \pm 1,115 \pm 1,127 \pm 1$, and $131 \pm 1 \mathrm{kDa}$, respectively (values expressed as the mean of different batches \pm standard deviation). The absolute values of peaks depended strictly on the amount of enzyme weighed, which might be subjected to slight variations between different experiments. Therefore, each peak was expressed as an Index (see Material and Methods section). Other small peaks (usually lower than 5\%) irregularly present in different batches, were considered as "remnants." Index values of remnants are inversely correlated with CII index values $(P<0.001, \mathrm{r}=-0.656)$. Liberase ${ }^{\mathrm{TM}}$ batches differ in terms of proportion of each peak (Table 1). Peak $B$ and peak $G$ significantly and inversely correlate $(P<0.001, r=-0.991$, Fig. $1 \mathrm{~b})$, suggesting that the smaller protein (peak G) could be derived from the larger one (peak B).

To identify the main peaks, we also performed a microelectrophoresis on the separate fractions produced by Roche. In Figure $2 \mathrm{a}-\mathrm{c}$, the profiles of the separated components Collagenase Class I (CI), Collagenase Class II (CII), and neutral protease (Thermolysin) are shown and compared with a Liberase $^{\mathrm{TM}}$ profile. CII enzyme was overlapping with peak D (molecular weight $128 \pm 2 \mathrm{kDa}$, Fig. $2 \mathrm{a}$ ), CI with peak B (molecular weight $131 \pm 1 \mathrm{kDa})$, and $\mathrm{G}$ (115 $\pm 1 \mathrm{kDa}$; Fig. 2b). Other small peaks with molecular weight $>34 \mathrm{kDa}$, irregularly present in different batches ( 10 of 15 batches, remnants), were present only in vials containing CII collagenase (Fig. 2a). Thermolysin profile showed few small peaks; that with the greatest proportion value (molecular weight $34 \pm 1$ ) was overlapping with peak $\mathrm{p}$ (Fig. 2c).

\section{Correlation Between Pealks and Isolation Outcome}

The number of samples was insufficient to allow a direct comparison among characteristics and results achieved using different Liberase lots (see Table 2). Therefore values of CI, CII, remnants, and protease peaks of different Liberase ${ }^{\mathrm{TM}}$ batches were pooled together and correlated with some representative parameters of isolation outcome: $\mathrm{NE}$, digestion time, purity.

In these analysis NE directly and significantly correlated with CII $(P<0.001, r=0.305$, Fig. 3a) and inversely, near to the significance, with remnants $(P=0.090, r=-0.133)$. Digestion time inversely correlated with CII (significance $P<0.001, \mathrm{r}=-0.502)$ and directly with remnants $(P=0.001$, $\mathrm{r}=0.266)$. The purity of preparations inversely and significantly correlated with CI (peak G) $(P=0.024, \mathrm{r}=-0.265)$ and directly with CI (peak B) $(P=0.028, r=0.260$, Fig. $3 b)$. No correlations were observed between protease peak values and isolation outcome (data not shown). As previously reported (4) there is a large dispersion of the data (and therefore a low
Analisys by microelectophoresis

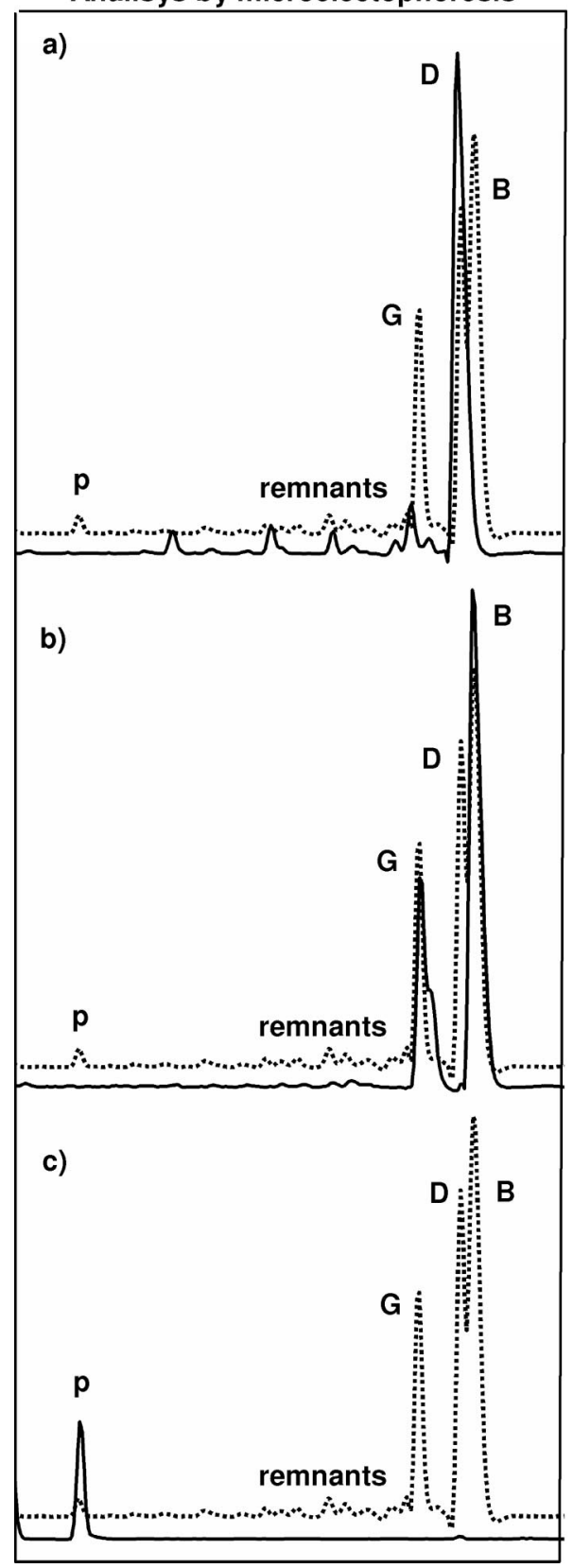

FIGURE 2. Characterization of Liberase components. (a-c) reported data achieved by microelectrophoresis technique. An example of peak profile of a Liberase ${ }^{\mathrm{TM}}$ batch was represented by dotted line. (a) The Liberase ${ }^{\mathrm{TM}}$ profile was superimposed with that of class II enzyme (CII, solid line) showing that CII correspond to peak D and to "remnants." (b) The Liberase ${ }^{\mathrm{TM}}$ profile was superimposed with that of class I enzyme (CI, solid line) showing that CI corresponded to peak $\mathrm{G}$ and B. (c) The Liberase ${ }^{\mathrm{TM}}$ profile was superimposed to Thermolysin (solid line) showing that thermolysin correspond to peak $\mathrm{p}$.

" $r$ " value) and this was expected according to the evidence that many variables are affecting human islet isolation yield. Because of the limited number of cases $(n=163)$ and to the large number of variables affecting islet isolation $(n=32$ in our previous study, plus these new 4 variables) we were un- 
TABLE 2. Isolation outcome according to different Liberase batches

\begin{tabular}{ccccc} 
Liberase $^{\mathrm{TM}}$ batches & Number of cases & Equivalent islet number \pm SD & Purity index \pm SD & Digestion time \pm SD (min) \\
\hline 93047420 & 45 & $172932 \pm 113972$ & $0.4 \pm 0.2$ & $21 \pm 6$ \\
90287220 & 24 & $149482 \pm 131464$ & $0.6 \pm 0.2$ & $25 \pm 4$ \\
93017120 & 10 & $85857 \pm 90580$ & $0.5 \pm 0.3$ & $23 \pm 6$ \\
93114320 & 15 & $177440 \pm 108760$ & $0.4 \pm 0.1$ & $22 \pm 8$ \\
93126020 & 4 & $272250 \pm 73477$ & $0.5 \pm 0.1$ & $22 \pm 5$ \\
93145320 & 1 & 396000 & 0.5 & 24 \\
93152720 & 2 & $67500 \pm 95459$ & $0.3 \pm 0.4$ & 19 \\
93028820 & 1 & 150000 & 0.6 & $25 \pm 6$ \\
93045820 & 15 & $130885 \pm 76700$ & $0.4 \pm 0.2$ & $28 \pm 10$ \\
93142020 & 2 & $96600 \pm 136613$ & $0.4 \pm 0.6$ & $16 \pm 5$ \\
93167720 & 8 & $228694 \pm 94309$ & $0.6 \pm 0.1$ & $12 \pm 3$ \\
93163920 & 18 & $279649 \pm 84516$ & $0.6 \pm 0.1$ & 27 \\
93187520 & 1 & 25000 & 0.4 & 10 \\
93172920 & 1 & 0 & Not assessable & $15 \pm 4$ \\
93273520 & 15 & $178287 \pm 112772$ & $0.7 \pm 0.02$ & \\
\hline
\end{tabular}

able to apply a proper multivariate analysis on these data. In a previous multivariate analysis on more than 400 isolations, we found that the activity/mg of collagenase corrected for the total $\mathrm{mg}$ of enzyme used in each isolation (total activity) strictly correlated with the number of isolated islets (4). In the present study we found that when CII is similarly corrected for the mg used in each isolation, it strictly correlates with total activity $(P<0.001, \mathrm{r}=0.796)$.

We previously proposed as threshold to define the success of islet isolation a procedure able to achieve islet yield $>250,000$ (equivalent islet) and with $>20 \%$ of purity (4). Accordingly, isolations were divided in two groups: successful and unsuccessful. A significant difference was observed between CII values of enzymes used in successful and unsuccessful islet isolation $(P=0.006$, Fig. $3 c)$. The results of statistical analyses did not change if only batches used more than three times were included in the study.

We observed no statistical correlations between CI or CII peak values and islet cell viability or insulin responsiveness to glucose (data not shown). However, the value of these statistical analyses was probably insufficient due to the poor number of cases available compare to the large dispersion of the data observed.

\section{Predictive Role of Collagenase Fractions Versus Transplantation}

High number of isolated islets, together with good islet purity and quality, are taken into account in order to define an islet preparation's suitability for transplantation. We verified the association of each component of Liberase ${ }^{\mathrm{TM}}$ with the decision as to whether or not to transplant a preparation. In isolations using a CII peak value $\geq 50$ percentile, 33 out of 98 were transplanted, and when values were $<50$ percentile, 9 of 65 were transplanted $\left(\chi^{2}\right.$-test, $\left.P=0.005\right)$. Considering that the purity of preparations, together with NE, is a prerequisite for islet transplantation, an additional criterion was introduced due to the suggested positive role of $\mathrm{CI}$ peak $\mathrm{B}$ on purification. In isolation using CII peak value $\geq 50$ percentile and CI peak B value $\geq 50$ percentile, 15 out of 36 were transplanted and in the remaining cases, 27 out of 127 were transplanted ( $\chi^{2}$-test, $\left.P=0.013\right)$.

From Figure 4, it appears evident that the highest values of CII and CI peak B correspond neither to the highest islet yield nor to the highest number of the transplanted islet preparations. Accordingly if CII peak index is limited to $0.294-$ 0.315 and $\mathrm{CI}$ peak B to $0.20-0.35$. (and as a consequence, the remaining enzyme, CI peak $\mathrm{G}$ in the range of $0.25-0.40$ ), 13 out 22 islet preparations were transplanted vs. 29 of 141 islet preparations in the other cases $(P<0.001)$.

In recipients receiving islet transplantation c-peptide values 1 week after transplantation correlated with CII peak values $(P=0.041, \mathrm{r}=0.652, \mathrm{n}=10)$ and $\mathrm{NE}(P=0.003$, $\mathrm{r}=0.830, \mathrm{n}=10)$. No relationships were observed between CI peak B or peak $G$ values with one week graft function. No correlation was found one month after transplantation between in vivo islet graft and Liberase peak values (data not shown).

\section{DISCUSSION}

The available information concerning the characteristics and composition of collagenase batches that are effective in the digestion of human pancreas for islet transplants is scarce and incomplete. Data achieved in animal model of pancreas digestion should not applied without criticisms in human pancreas where the clinical condition of the donors and therefore of pancreas heavily interfere with digestion success rate. In this work, we evaluated the components of Liberase $^{\mathrm{TM}}$ blend enzyme and their functional role in digestion of human pancreas. This information represents the real basis for a further standardization of the procedures. The issue of standardization of the procedures is not only technical. The discontinuity of isolation success is one of the most important factors that limit the clinical applicability of islet transplantation. Discontinuous isolation results means in fact unpredictable costs of procedures and high variability of islet quality and, finally, the impossibility to perform large studies aimed at improving graft function. 

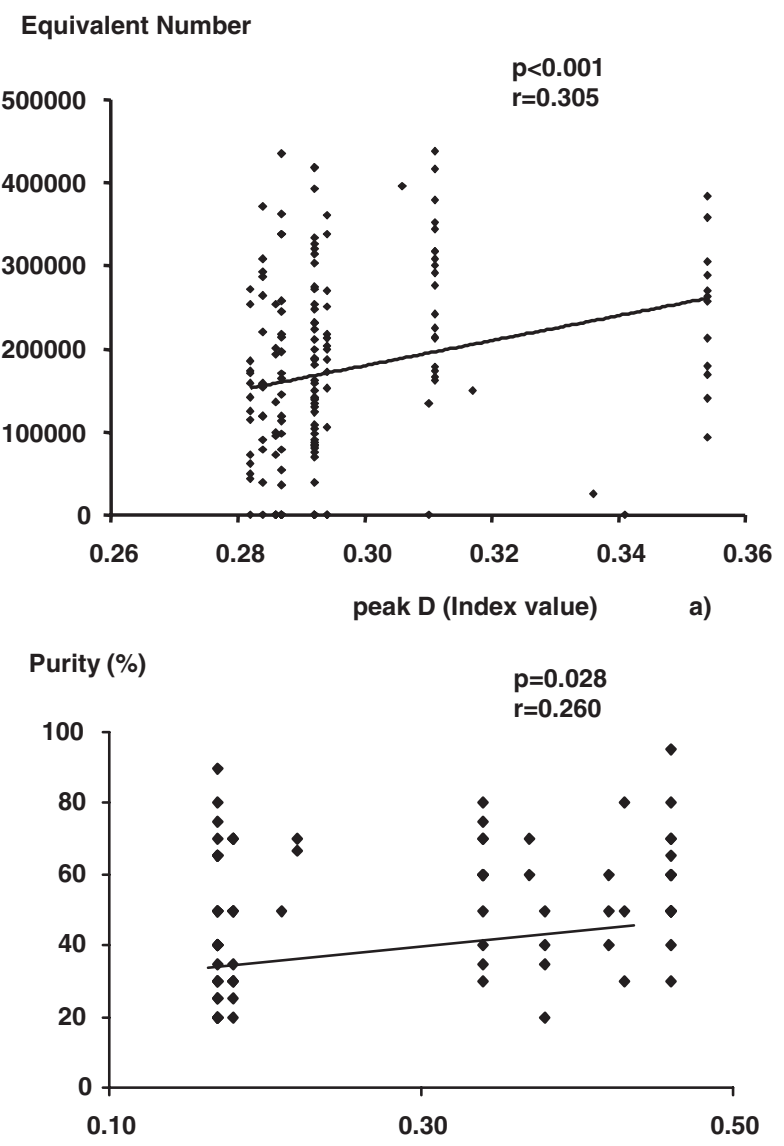

b)

peak B (Index value)

peak D (Index value)

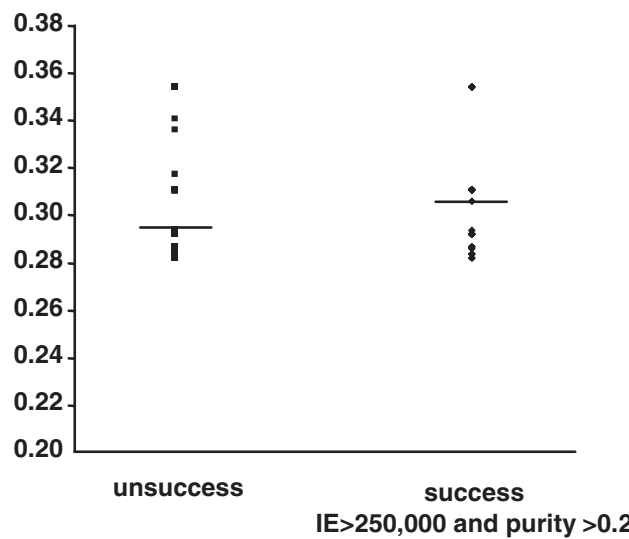

c)

FIGURE 3. Correlation between $D$ indexes of different Liberase ${ }^{\mathrm{TM}}$ batches and the number of equivalent islets (NE) (a) and correlation between B indexes and islet purity (b) of the corresponding isolations in which these enzymes were used. (c) Represents the comparison between D indexes of enzyme of successful vs. failed isolation. Black bar indicates the mean of $\mathrm{D}$ index values.

We observed that collagenase class II was shown to be the most important enzyme for successful islet yield, thus confirming in human pancreas digestion what was demonstrated in animal model of pancreatic isolation (16). Indeed
Equivalent Number
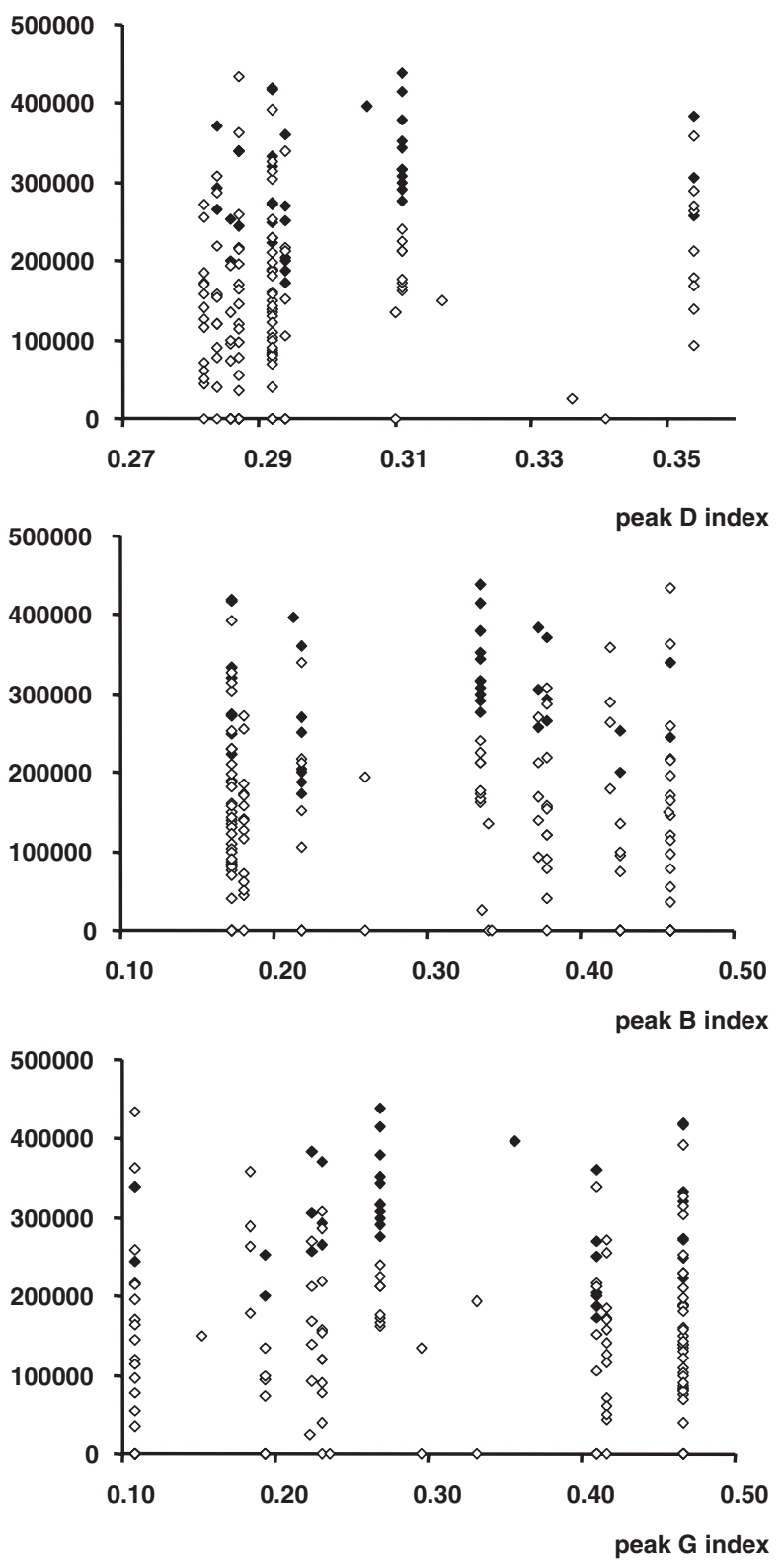

FIGURE 4. Correlation between peak indexes and the decision of transplanting islet preparation. Correlation between D (a), B (b), and G (c) indexes obtained by microelectrophoresis analysis and the number of equivalent islets (NE) of the corresponding isolations in which these enzymes were used. Black squares indicate transplanted preparations, white squares indicate not transplanted preparations.

this is the only class whose index values within the enzyme blend directly correlates with the number of isolated islets. Thus, CII is associated also with the decision as to whether or not to transplant a preparation or not. This was quite an unexpected result, because of the limited range of variability (28-35\%) between different batches, as previously reported (3). Considering the direct correlation between NE and the CII proportion in a vial, it would be expected that the highest 
islet yield as well as the highest number of transplanted preparations would be obtained with the highest CII values. From Figure 3 and Figure 4, it appears that this does not apply as the best results in terms of transplant occur with $0.294-0.315$ CII index values, a very narrow range.

The purified CII fraction collagenase shows some protein components present in small amounts (about 20\% of enzyme blend), and absent in CI fraction collagenase. The presence of these proteins is inversely correlated with the presence of CII collagenase $(P<0.001, \mathrm{r}=-0.656)$, which suggests that they may result from its degradation. The proportion of fragments seems to have a negative role in the isolation process (although the results are not statistically significant) thus suggesting that the quality and the purity of CII collagenase should be further improved.

It is clear that the success of isolation with regard to number of isolated islets depends on many variables, including donor characteristics and isolation procedures (4). Therefore one component of enzyme should not determine per se the fate of isolation. This appears also by the low value $(r=0.305)$, albeit statistically significant, of the correlation between collagenase class II index and number of isolated islets in our retrospective analysis. Correct analyses of an eventual association between variables of isolation should be multivariate in a large number of cases, and this was not the case of this study, conducted on 163 isolations. In a recent retrospective multivariate analysis on 32 variables conducted on more than 400 isolations, we demonstrated that the collagenase activity, normalized to the amount of the enzyme used in the isolation (total activity), is one of the most important variables associated with the number of islets obtained (4). CII proportion similarly normalized to the milligram of enzyme used in each isolation, strictly correlated with the total activity with $r=0.796$. Therefore, these two parameters appear concordant, allowing us to hypothesize that the relation between CII enzyme and the number of isolated islets observed may be significant also in a multivariate analysis on a large number of isolations although, of course, this should be further confirmed.

The increased islet yield and purity achieved with an optimal collagenase blend could be of relatively minor relevance if associated with a reduced islet function or survival. No correlation was observed between enzyme characteristics and islet viability and insulin secretion, but the large data dispersion at this regard and the poor number of cases available for this statistics limited the value of the analyses. We used 2 additional indirect parameters for evaluation of the quality of isolated islets: the suitability of an islet preparation for transplantation and in vivo graft function. Islet preparation is usually defined transplantable if it is adequate in terms of islet mass, viability and purity. Therefore, it should be considered per se an overall indirect parameter of the quality, and this parameter was associated with high CII index values, too. In vivo graft function should be not recognized as an appropriate parameter for our study, being that in vivo graft function involves too many variables, although-also in this case-a significant correlation was observed between CII and early graft function. At this regard, we do not believe CII can directly affect in vivo graft function, or at least the role of CII in affecting graft function should be secondary to the larger transplanted islet mass made available using a successful enzyme.

CI collagenase, another essential component of a blend enzyme designated to digest human pancreas, increases digestion rate as reported (16). In our blend enzyme for islet isolation we identified two different components of CI collagenase (designated as peak B and G), which are inversely correlated. Although neither of these correlates with the number of isolated islets, peak B correlates directly with the purity of the preparation. It was already reported that enzymes used for pancreas digestion affected islet purification (21-22). Mechanisms should include the duration of digestion (and therefore of warm ischemia cell exposure), cell death, incomplete tissue digestion that collectively could contribute to exocrine cell degranulation and damage resulting in variable purification of islet preparation. However, the best results concerning the number of isolated islets and suitability of the preparation for transplant are obtained from a mixture of CI components. From this point of view, our conclusion differs from that reported by Lakey on the analysis of Liberase ${ }^{\mathrm{TM}}$ batch by highperformance liquid chromatography $(3,25)$. In fact, Lakey describes two components of CI collagenase, of which only one was associated (in a small number of cases) with isolation success (3). It should be noted that Lakey et al. had the possibility of using batch enzymes containing only one component of class I collagenase $(3,22)$. We expect in the future to test batches with similar characteristics in our laboratory.

From our analyses, it appeared that the small differences in neutral proteases content between different Liberase batches failed to significantly affect pancreas digestion outcome, differently from what it was recently reported (25). These enzymes are considered important in the early steps of digestion (21) and the composition and content of neutral protease present in our Liberase batches appeared to be adequate for a successful digestion. Further studies should also be necessary to fully analyze the molecular structure of these enzymes.

We confirmed differences between vials as reported by Lakey, although to a lesser extent (3). In this regard, we did not consider mass and enzyme activity changes in each vial, thus probably underestimating the real variability among vials. The microelectrophoresis analysis appears simple and reproducible and could therefore be applied to a selection of successful batches for the isolation. In fact, compared with high-performance liquid chromatography, it is quicker, describing enzymatic blend composition in few minutes.

In conclusion, the significance of this study lies in the fact that it represents the characterization of the components of blended enzymes designated for human isolations. This represents a first step toward the production of enzymes using recombinant techniques, the only means of guaranteeing continuous and reproducible isolation results. In addition the standardization of enzymes for digestion of human pancreas should promote the digestion success rate of other tissue. However, a more standardized production (3) capable of guaranteeing the stability and reproducibility of the enzyme for isolation purpose is now needed.

\section{ACIKNOWLEDGMENTS}

This work was supported by grants from the Juvenile Diabetes Research Foundation (5-2001-172, 1-2000-780, JT-01) 
and from Telethon Italy (JT-01). Finally, we also thank Ezio Bonifacio for his critical suggestions on the manuscript and Danilo Romeres for the careful English revision of the text.

\section{REFERENCES}

1. Ricordi C, Lakey JR, Hering BJ. Challenges toward standardization of islet isolation technology. Transplant Proc 2001; 33: 1709.

2. Brandhorst $H$, Brandhorst D, Hering BJ, Bretzel RG. Significant progress in porcine islet mass isolation utilizing liberase HI for enzymatic low-temperature pancreas digestion. Transplantation 1999; 68: 355.

3. Barnett MJ, Zhai X, LeGatt DF, Cheng SB, Shapiro AM, Lakey JR. Quantitative assessment of collagenase blends for human islet isolation. Transplantation 2005; 80: 723.

4. R Nano, B Clissi, R Melzi, G, et al. Islet isolation for allotransplantation: Variables associated with successful islet yield and graft function. Diabetologia 2005; 48: 906.

5. Linetsky E, Bottino R, Lehmann R, Alejandro R, Inverardi L, Ricordi C. Improved human islet isolation using a new enzyme blend, Liberase. Diabetes 1997; 46: 1120.

6. Bucher P, Mathe Z, Morel P, et al. Assessment of a novel two-component enzyme preparation for human islet isolation and transplantation. Transplantation 2005; 79: 91

7. Brandhorst H, Brandhorst D, Hesse F, et al. Successful human islet isolation utilizing recombinant collagenase. Diabetes 2003; 52: 1143.

8. Hughes SJ, Clark A, McShane P, Contractor HH, Gray DW, Johnson PR. Characterisation of collagen VI within the islet-exocrine interface of the human pancreas: Implications for clinical islet isolation? Transplantation 2006; 81: 423.

9. Yoshida E, Noda H. Isolation and characterization of collagenases I and II from Clostridium histolyticum. Biochim Biophys Acta 1965; 1965; 105: 562.

10. Kono T. Purification and partial characterization of collagenolytic enzymes from Clostridium histolyticum. Biochemistry 1968; 7: 1106

11. Bond MD, Van Wart HE. Characterization of the individual collagenases from Clostridium histolyticum. Biochemistry 1984; 23: 3085

12. Bond MD, Van Wart HE. Relationship between the individual collagenases of Clostridium histolyticum: Evidence for evolution by gene duplication. Biochemistry 1984; 23: 3092.

13. Mallya S, Mookhtiar K, Van Wart H. Kinetics of hydrolysis of type I, II, III collagens by the class I and II Clostridium histolyticum collagenases. J Protein Chem 1992; 11: 99.
14. Mookhtiar K, Steinbrink R, Van Wart H. Mode of hydrolysis of collagenlike peptides by class I and class II Clostridium histolyticum collagenases: Evidence for both endopeptidase and tripeptidylcarboxypeptidase activities. Biochemistry 1985; 24: 6527.

15. Van Wart H, Steinbrink DR. Complementary substrate specificities of class I and class II collagenases from Clostridium histolyticum. Biochemistry 1985; 24: 6520.

16. Wolters GHJ, Vos-Scheperkeuter GH, Hun-Chi Lin, van Schilfgaarde R. Different roles of class I and class II Clostridium histolyticum collagenase in rat pancreatic islet isolation. Diabetes 1995; 44: 227.

17. Vos-Scheperkeuter GH, van Suylichem PT, Vonk MW, Wolters GH, van Schilfgaarde R. Histochemical analysis of the role of class I and class II Clostridium histolyticum collagenase in the degradation of rat pancreatic extracellular matrix for islet isolation. Cell Transplant 1997; 6: 403.

18. Brandhorst D, Huettler S, Alt A, Raemsch-Guenther N, Kurfuerst M, Bretzel RG, Brandhorst $\mathrm{H}$. Adjustment of the ratio between collagenase class II and I improves islet isolation outcome. Transplant Proc 2005; 37: 3450 .

19. Wolters GH, Vos-Scheperkeuter GH, Van Deijnen JH, van Schilgaarde $\mathrm{R}$. An analysis of the role of collagenase and protease in the enzymatic dissociation of the rat pancreas for islet isolation. Diabetologia 1992; 35: 735.

20. Bond M, Van Wart H. Purification and separation of individual collagenases of Clostridium histolyticum using red dye ligand chromatography. Biochemistry 1984; 23: 3077.

21. Brandhorst H, Brendel MD, Eckhard M, Bretzel RG, Brandhorst D. Influence of neutral protease activity on human islet isolation outcome. Transplant Proc 2005; 37: 241.

22. Brandhorst H, Alt A, Huettler S, Raemsch-Guenther N, Kurfuerst M, Bretzel RG, Brandhorst D. The ratio between class II and class I collagenase determines the amount of neutral protease activity required for efficient islet release from the rat pancreas. Transplant Proc 2005; 37: 215.

23. Ricordi C, Lacy PE, Scharp DW. Automated islet isolation from human pancreas. Diabetes 1988; 38 Suppl 1: 140.

24. Vargas F, Vives-Pi M, Somoza N, et al. Advantages of using a cell separator and metrizamide gradients for human islet purification. Transplantation 1996; 61: 1562.

25. Kin T, Zhai X, Murdoch TB, Salam A, Shapiro AM, Lakey JR. Enhancing the success of human islet isolation through optimization and characterization of pancreas dissociation enzyme. Am J Transplant 2007; 7: 1233 